\title{
Gambling in Canada During the COVID Lockdown: Prospective National Survey
}

\author{
Carrie A. Shaw ${ }^{1,2}$ D . David C. Hodgins ${ }^{3}$ (D) Robert J. Williams ${ }^{2}$ (D) Yale D. Belanger $^{2}$ (D) \\ Darren R. Christensen ${ }^{2}$ (D) Nady el-Guebaly ${ }^{3}$ - Daniel S. McGrath ${ }^{3}$. \\ Fiona Nicoll ${ }^{4}$. Garry J. Smith ${ }^{4} \cdot$ Rhys M. G. Stevens ${ }^{2} \mathbb{D}$
}

Accepted: 3 September 2021 / Published online: 20 September 2021

(c) The Author(s), under exclusive licence to Springer Science+Business Media, LLC, part of Springer Nature 2021

\begin{abstract}
The current study investigated the impact of the COVID pandemic lockdown on gambling and problem gambling in Canada. The AGRI National Project's online panel participants $(N=3449)$ provided baseline gambling data 6 months prior to the pandemic. Re-surveying this sample during the lockdown provided an opportunity to make quantitative comparisons of the changes. Nearly one-third of gamblers reported ceasing gambling altogether during the lockdown. For the continuing gamblers, quantitative data indicated significant decreases in gambling frequency, time spent in gambling sessions, money spent, and the number of game types played. Qualitative perceptions of changes in gambling were examined and the accuracy of these reports were not closely aligned with actual changes in gambling. Gambling platform was the only gambling engagement metric where increases were found with $\sim 17 \%$ of the gambling sample migrating to online gambling during the lockdown. Although problem gambling within the sample generally declined, consistent with previous literature, it was also found that gambling online-among other biopsychosocial factors-was a significant predictor for classification as a problem gambler during the lockdown. COVID-specific influences on health, employment, leisure time and social isolation were moderately associated with problem gambling scores but were not independent predictors of changes in gambling engagement during lockdown. Future studies are required to assess if the pandemic related changes in gambling evidenced in this study remain stable, or if engagement reverts to pre-pandemic levels when the pandemic response allows for the re-opening of land-based gambling venues.
\end{abstract}

Keywords Gambling $\cdot$ Problem gambling $\cdot$ Online gambling $\cdot$ COVID $\cdot$ Pandemic

Public Health Significance: This study demonstrated that the impacts of the COVID pandemic lockdown on gambling in Canada were heterogeneous, with some individuals abstaining during the lockdown and others diversifying their gambling repertoire. The variability of engagement indicates that there is need for continued monitoring with the goal of assessing the stability of these changes. This study also highlights the importance of evaluating gambling changes across time and using validated quantitative metrics.

Carrie A. Shaw

carrieann.shaw1@gmail.com

Extended author information available on the last page of the article 


\section{Introduction}

The novel coronavirus (COVID) pandemic has had a profound impact on the lives of people worldwide. The impact includes severe health, social, emotional, and economic effects. In many jurisdictions, including Canada, "social distancing" (Abel \& McQueen, 2020) is strongly promoted and entails creating and keeping safe distances between individuals, as well as reducing the number of times individuals come into close contact with others. Included in the pandemic response strategy is the closure or "lockdown" of retail operations, educational institutions, and cultural, sports and entertainment-related venues (e.g., casinos, clubs, theaters, museums, sporting arenas). Many individuals are currently, or have been, working remotely when possible while others have had their employment hours reduced or eliminated. Individuals who are infected, or possibly infected, with the virus are required to self-isolate for 10-14 days.

The pandemic response strategy has significantly impacted the availability of gambling. In all Canadian provinces, all land-based gambling venues temporarily closed in March 2020, including casinos, bingo halls, horse racing venues, and other locations that offer slot machines and video lottery terminals (VLTs) (Czegledy, 2020). Online gambling opportunities continued to be available, however, including lottery ticket purchases and online gambling site offerings. In Canada, in early 2020, 8 out of 10 provinces offered provincially operated online gambling sites providing a variety of types of gambling. Canadians are also able to access out-of-country online gambling sites.

Speculation about the consequences of restriction of gambling opportunities has appeared in the media and in peer reviewed commentaries. First, the economic impact of the lock-down on the gambling industry has been highlighted, both in the dramatic reduction of land-based gambling revenues and, in contrast, reports of an increase in online gambling activity and profits (e.g., Howie, 2020; Shivdas, 2020). There is also speculation about the impact of the loss of gambling opportunities on individuals who are suffering gambling-related problems (Marsden et al., 2020). Some have suggested that reduction of gambling availability may reduce gambling-related problems by providing people with the opportunity to curtail their gambling (e.g., Elder, 2020). In support of this, calls to a provincial gambling helpline in Canada dropped after casinos closed (Turner, 2020). Others note the potential of increased problems as people migrate to online gambling (e.g., Håkansson et al., 2020; Horner, 2020; Xuereb et al., 2020). The concern about migration to online gambling is that online gambling is associated with greater prevalence of gamblingrelated problems, in large part because of its greater convenience, 24-h access, ability to play when intoxicated, the solitary nature of play, and because it is more difficult for online problem gamblers to avoid temptation (i.e., it is easier for a land-based problem gambler to avoid land-based casinos, racetracks and bingo halls than it is for an online problem gambler to avoid using computers or the Internet) (Brunelle et al., 2012; Wood \& Williams, 2011).

Finally, some have predicted that the stress, loneliness, unemployment, and job insecurity related to the pandemic might promote relapse in people recovering from gambling problems (Campbell, 2020; Jones, 2020) or alternatively, gambling initiation or exacerbation (van Schalkwyk et al., 2020). This latter impact was noted in Greece (Economou et al., 2019) and Iceland (Olason et al., 2017) where prevalence of gambling rose after financial crises.

An editorial in Addiction underscored the urgency of research and surveillance of the pandemic effects on addictive behaviors (Marsden et al., 2020). A recent review 
found 11 published and unpublished cross sectional surveys in which participants were asked to compare their gambling prior to and during the COVID lockdown (Hodgins \& Stevens, 2021). These surveys report overall reduction in gambling in their samples but also that a small subpopulation described increased gambling.

A major limitation of this research is that it relies on participants providing an appraisal of the change in their gambling related to COVID rather than a comparison of gambling reported earlier to current gambling. These unvalidated measures of perceived change are likely of limited reliability. Moreover, measures of overall gambling are significantly less reliable than measures aggregated across reports of engagement in specific gambling activities (Williams et al., 2017).

Very few longitudinal studies exist (Hodgins \& Stevens, 2021). In one peer reviewed article, behavioral data from sports bettors in Europe indicated that these bettors did not switch to more/different online games during lockdown and that there was a significant decrease in overall money wagered (Auer et al., 2020). Similarly, in a UK survey (Gunstrone et al., 2020) that re-assessed a sample of individuals who had experienced some gambling related harm, or had family members who had experienced harm, gamblers with greater problem severity and younger gamblers were more likely to report that their gambling had increased in frequency and expenditure than other respondents. The study did not report individual changes in actual gambling frequency and expenditure between the two assessments, but overall gambling decreased significantly. Participation in all types of gambling decreased, except for online casino games, which increased slightly.

In another UK study (Fluharty et al., 2020), a variety of methods were used to recruit a large sample of adults who provided weekly assessments of gambling starting during the lockdown period. Most of the sample reported no change in their gambling (79\%), 11 decreased and $9 \%$ increased. Although problem gambling was not assessed, individuals increasing gambling also reported boredom, generalized anxiety and major depression.

An Australian online panel study of the general population (Biddle, 2020) reported that overall gambling and problem gambling declined with the largest decrease for informal games, bingo and casino games. Gambling during the pandemic was associated with a positive change in life satisfaction and problem gambling declined even among individuals who continued to gamble.

The existing cross sectional and longitudinal research suggests that the impacts of the COVID pandemic, including the lockdown, are likely complex and variable (Hodgins $\&$ Stevens, 2021). The present study adds to the small prospective literature with a large national baseline of gambling behavior collected before the pandemic and a reassessment of this behavior during the lockdown. The study goals were to:

Describe the change in individual gambling during the COVID lockdown compared with gambling in the previous year. This included the examination of migration to online gambling and the impact on problem gambling. [G1]

Describe gamblers perceptions of whether their gambling frequency and expenditures changed from typical pre-pandemic monthly gambling to that during the lockdown and to assess quantifiable changes in gambling as a function of these perceived changes.

\section{[G2]}

Examine the cross-sectional characteristics of individuals who increased versus decreased gambling during lockdown. [G3]

Examine the predictors of problem gambling during the pandemic lockdown in a longitudinal dataset. [G4] 


\section{Method}

The data used in this report were collected as a supplement to the AGRI National Project (ANP). ANP Project details, including the full survey, can be seen at: https://www.ucalg ary.ca/research/national-gambling-study/. This study was approved by the University of Lethbridge Human Ethics Review Board (Protocol\#: 2018-063).

\section{Sample}

The ANP Online Panel sample was recruited from the Leger Opinion (LEO) registered pool of online panelists, a panel pool that is structured to be geographically and demographically representative of the adult $(18+)$ population in Canada. Between August 16 to October 10, 2018, members of LEO Leger who reported gambling at least once per month in the past year were directed to an online gambling survey. Recruitment continued until at least 10,000 surveys were completed by an equal number of respondents per province or region. In the end, a Baseline sample of 10,199 was obtained. Between August 20 and November 30, 2019, ANP Online Panelists were re-contacted and asked to take a follow-up survey. A total of 4,707 completed the follow-up survey, which represents $82.50 \%$ of those who had agreed to be re-contacted after the Baseline ANP survey, and $46.15 \%$ of the Baseline ANP participants.

The information provided by participants during the ANP follow-up assessment was used as the Pre-COVID baseline data for the present COVID panel study. Pre-COVID baseline participants were re-contacted with a request to complete a 15-min COVID-Lockdown survey, with data collection taking place online between May 14 and June 1, 2020. ${ }^{1}$ Respondents received \$10 CAD plus the standard LEO remuneration. In total, 3449 people completed the COVID-Lockdown survey, which represents a $72.2 \%$ retention rate from the Pre-COVID baseline.

\section{Measures}

For each construct, a marker [G\#] is provided to indicate what study goal the construct was used to address. In addition to collecting demographic data, used for sample description and [G3, G4], the Pre-COVID baseline and COVID-Lockdown surveys assessed the constructs described below.

\section{Comorbidities}

Past month use of tobacco, alcohol, cannabis, and illicit drugs; spending on cannabis; alcohol and drug use help seeking behaviors; and past month behavioral addictions. In addition, past year substance use disorder, post-traumatic stress, generalized anxiety, panic disorder and major depression were assessed using DSM-5 criteria (American Psychiatric Association et al., 2013). [G3, G4]

\footnotetext{
${ }^{1}$ This was prior to the re-opening of any Canadian establishment that could provide legal in person gambling experiences.
} 
The current study also made use of the Pre-COVID baseline information for past year level of stress, an item adopted from the publicly available annual Canadian Community Health Survey (CCHS item GEN_Q020), with anchor points $1=$ Not at all stressful to 5=Extremely stressful. The COVID-Lockdown survey used the CCHS level of stress item to assess level of stress in the past month since the onset of the pandemic with the same response options. In addition, the COVID-Lockdown survey included items assessing past month (since the onset of the pandemic) leisure time and social interaction. For each of these constructs, participants were asked: "Thinking about the amount of in your life, since the onset of COVID-19, has this increased, decreased or stayed the same?" with response options for each item being $1=$ Increased significantly, $2=$ Increased somewhat, $3=$ no change, $4=$ Decreased somewhat, and $5=$ Decreased significantly.

\section{Personality}

Impulsivity was assessed in the Pre-COVID baseline survey using the NEO Personality Inventory-Revised (NEO PI-R) (Costa \& McCrae, 1992). Internal reliability of the NEO-PI-R domain scores are known to be high, ranging from 0.86 to 0.92 (Costa \& McCrae, 1992). The concurrent and discriminant validity of the NEO has been well established in both normal and clinical populations (Costa \& McCrae, 1992). [G3, G4]

\section{Gambling}

\section{Gambling Participation}

The Gambling Participation Instrument, GPI (Williams et al., 2017) assesses past year gambling participation for all of the primary dimensions of gambling: type, means of access, frequency, time, and expenditure (with time and expenditure being assessed by asking about 'typical month' time/spending in the past 12 months). The test-retest reliability coefficients of the GPI are fair to excellent, ranging from 0.46 to 0.84 , and the validity coefficients are good to excellent, ranging from 0.60 to 0.91 (Williams et al., 2017). The GPI was modified for the COVID-Lockdown survey to address the past month participation, so as to capture behavior specifically during the lockdown. The GPI also was modified to eliminate questions about participation in speculative financial activities (e.g., day trading) due to the volatile market fluctuations that occurred during the lockdown. The current study evaluated the following GPI gambling constructs (Williams et al., 2017): [G1, G2, G3, G4]

\section{Types}

Types of gambling is the total number of different gambling formats engaged in during the past month (i.e., Gambling types=lottery/raffle tickets, instant win tickets, electronic gambling machines (EGMs), casino table games, sports betting (includes horse racing), bingo, and 'other' types not listed). Minimum possible $=0$ and maximum possible $=7$.

\section{Frequency}

For each type of gambling, participants were asked to indicate how often in the past month they engaged in that gambling format. Response options were: $0=$ Never, $1=$ Less 
than once a month, $2=$ Once a month, $3=$ Two to three times a month, $4=$ Once a week, $5=$ Several times a week, $6=4$ or more times a week. The total combined frequency of gambling on all reported types of gambling then was summed, yielding a range of $0-48$.

\section{Net Expenditure}

Net expenditure (wins minus losses) was reported for each gambling type.

\section{Total Losses}

The net expenditures reported for each type of gambling were summed. Total losses then recoded any reported total wins to +1 so as to improve validity of expenditure estimates (Williams et al., 2017; Wood \& Williams, 2007).

\section{Time}

The sum of all time spent on all types of gambling, with values at and above 500 being reset to a maximum of $16 \mathrm{~h}$ per day for 31 days.

\section{Platform}

Derived from respondents reports of their means of access for each type of gambling game (e.g., bought a lottery ticket at a local store, played poker online). Responses were coded to indicate access to gambling by way of land-based only platform(s) or online platform.

\section{Subjective Perception of Change}

In addition to the GPI items, questions were included to assess the respondent's subjective perception of changes, if any, to gambling participation since physical distancing measures were instituted in March 2020. Specifically, participants were asked if-for each type of gambling - their frequency of engagement and expenditures had increased significantly, increased somewhat, decreased significantly, decreased somewhat, or not changed. [G2]

\section{Gambling Fallacies}

The Gambling Fallacies Measure (GFM) (Leonard Shaw et al., 2015). The GFM is a 10 -item multiple-choice instrument. High scores $($ maximum $=10)$ indicate greater resistance to gambling fallacies, and low scores indicate endorsement thereof (minimum $=0)$. The internal consistency of this instrument is very good (omega $=0.89$ ) (Leonard Shaw et al., 2020). The overall one-month test-retest reliability of the instrument is good (0.70).

\section{Past Year Problem Gambling}

The 'past 12 months' time frame was used in both the COVID-Lockdown and the PreCOVID baseline surveys allowing for direct comparisons. [G3, G4] 


\section{Problem Gambling Severity Index (PGSI) (Ferris \& Wynne, 2001)}

The 9 item PGSI provides a summed total score indicating past year severity as well as interpretive categories, $0=$ non-problem gambling, scores of $1-2=$ low risk, scores of 3-7=moderate risk, and scores of, 8 or higher $=$ problem gambling (Ferris \& Wynne, 2001). [G1, G2, G3, G4]

\section{COVID History}

A short series of questions was included that were adapted from the Epidemic - Pandemic Impacts Inventory (Grasso et al., 2020). These questions ask participants about themselves and about those living in the respondent's home regarding virus symptoms and testing, whether medical treatment was required due to COVID, if a hospital stay was required due to COVID, and whether someone died due to COVID (in the respondent's home or a close friend or family member out of home). From these questions two variables were created, one to indicate the respondents' personal health had been impacted by COVID and one to indicate the respondents' life had been impacted by COVID through close others' whose health had been impacted. [G3, G4].

\section{Analysis Plan}

\section{Data Handling}

The survey administration required a participant response for each applicable item and therefore sample size differences are due largely to the non-applicability of items for some respondents. Cases with non-applicable responses were excluded from analyses. All variables were checked for skewness (skew $\geq \pm 0.4$ ) and univariate outliers $(z> \pm 3.29$ ). Skew was detected in the gambling engagement variables time spent and money spent (total losses) and outliers were detected in the total gambling loss variables at both time points. Skew was not corrected by transformation, so therefore, the total losses variables for both time points were winsorized to mitigate the impact of outliers and non-parametric tests were used where possible. Because of the multiple planned analysis necessary to address the study aims and their exploratory nature, alpha level was set at 0.01 a priori with Bonferroni corrections for multiple comparisons used where appropriate. Also, in recognition of the difference between statistical and clinical significance, any associations at or below 0.17 are interpreted as weak (Hemphill, 2003).

\section{Analysis}

All analyses were completed using SPSS version 25. To compare change in individual gambling during the lockdown versus typical monthly gambling [G1], paired t-tests were calculated for continuous measures and non-parametric tests (i.e., McNemar) for categorical variables. Despite the skew in gambling engagement measures, paired t-tests were used for these analyses because these tests are robust against skew with large samples. Kruskal-Wallis tests with Bonferroni corrected post hoc Mann-Whitney U tests were used 
to compare individuals' perception of change in gambling engagement with their change from pre-Covid to lockdown. [G2]. The cross-sectional characteristic profiles of individuals reporting increases versus decreases in expenditures and frequency [G3] were examined using binary logical regression. Then a General Estimating Equation (GEE) was used to assess the relative predictive ability of variables of interest, including time, on problem gambler category [G4]. Individual characteristic variables significantly associated with our dependent variables were included in the cross-sectional predictive model (goal 3), and variables that were both, significant and moderate to strong correlates of increased problem gambling category, were included in our longitudinal analysis (GEE).

Prior to completing analyses to address the aims of the current study, logistic regression was used to investigate characteristics that differentiated Pre-COVID respondents who completed the COVID survey $(n=3449)$ from those who did not $(n=1258)$. All demographic and problem gambling variables examined in this study were included, and variable entry was forward stepwise. Variable entry order was determined by Wald statistic with a minimum entry of $p=0.10$ and removal level of $p=0.15$, with a cut point of 0.75 . Optimal model fit occurred with only four variables: sex, age, marital status, and household income. Individuals who did not complete the COVID-lockdown survey were more likely to be younger, be female, have higher income, and have had a marital status of separated (but still legally married) or widowed. A test of the full model against a constant only model was significant, $\chi^{2}(12, N=4707)=106.72, p<0.001$. The variance accounted for was moderate, Nagelkerke $\mathrm{R}$ squared $=0.03$, and the overall prediction success was also moderate $(53.40 \%)$ with $49.70 \%$ of completers successfully classified correctly and $63.30 \%$ of non-completers correctly classified. The interpretation of all findings should take these group differences into account.

\section{Results}

Table 1 presents the descriptive statistics for demographic and other study variables for the Pre-COVID and COVID-Lockdown data collection periods.

\section{Changes in Gambling Involvement During Lockdown [G1]}

The majority of individuals maintained their pre-pandemic gambling status with $69.40 \%$ $(n=2394)$ of individuals who were gamblers pre-COVID continuing to gamble during the lockdown, and $1.56 \%(n=54)$ of the sample remaining non-gamblers. That said, $28.47 \%$ $(n=982)$ ceased gambling during the lockdown, and $0.55 \%(n=19)$ began gambling during the lockdown.

Respondents' gambling platform at each data collection point was coded to indicate 'Land-based only' or 'Online' gambling (see Table 1). Of interest, 17.60\% ( $n=490)$ of Land-based only gamblers (Pre-COVID) were found to migrate to Online gambling during the lockdown period, which is a significant change, McNemar $=260.00, p<0.001$. Together with those who had previously engaged in, and continued to engage in, online gambling, a total of 894 gamblers (37\% of gamblers) reported online gambling.

Not surprisingly, given the widespread closure of land-based gambling venues, the mean number of gambling types engaged in during lockdown was significantly lower than the number of types reported for a typical month recorded six months earlier $\left(t_{(3371)}=-54.56, p=0.000, M_{D}=-1.23,95 \%\right.$ CI $\left.[-1.27,-1.19], d=0.94\right)$. Also, compared 


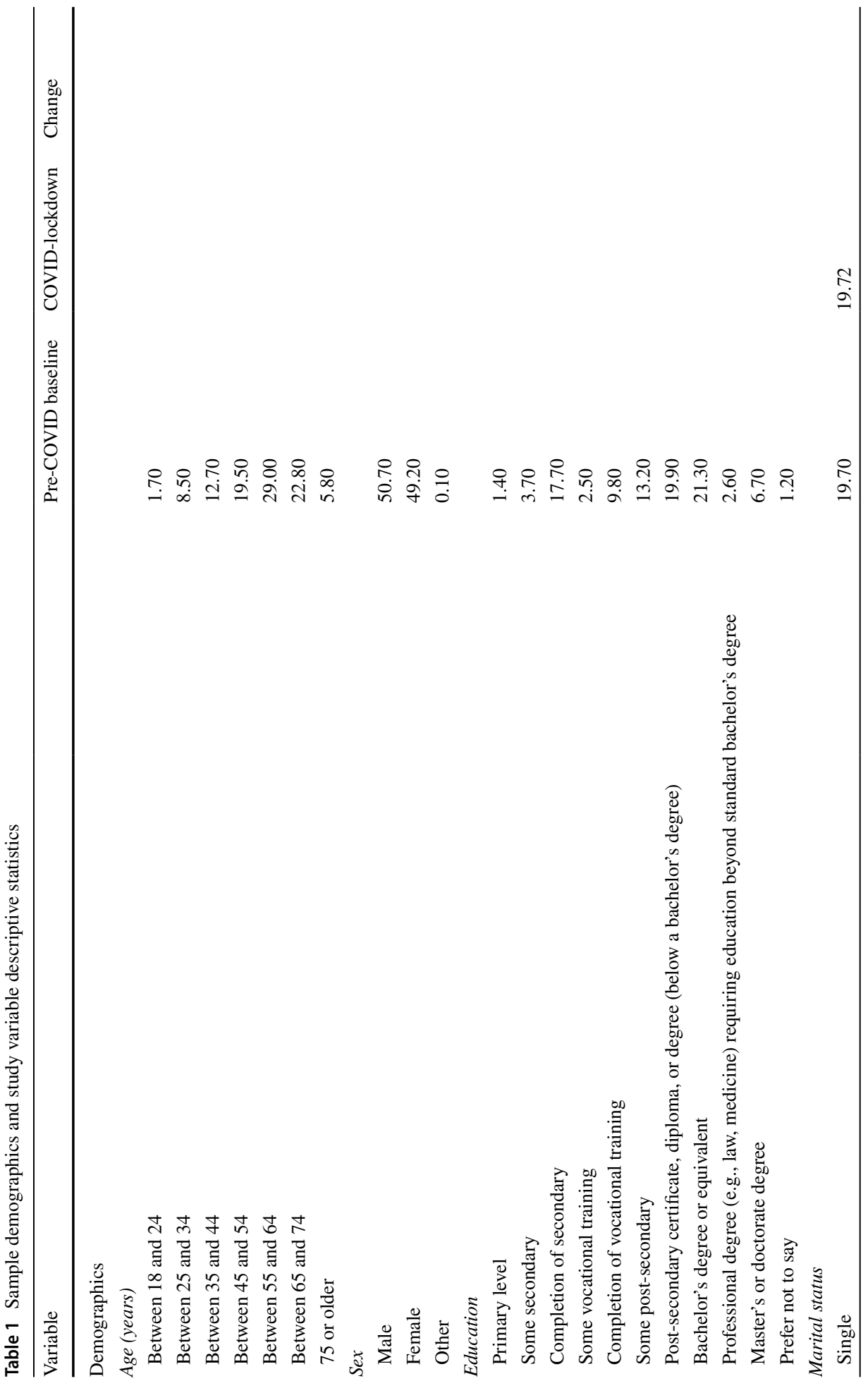




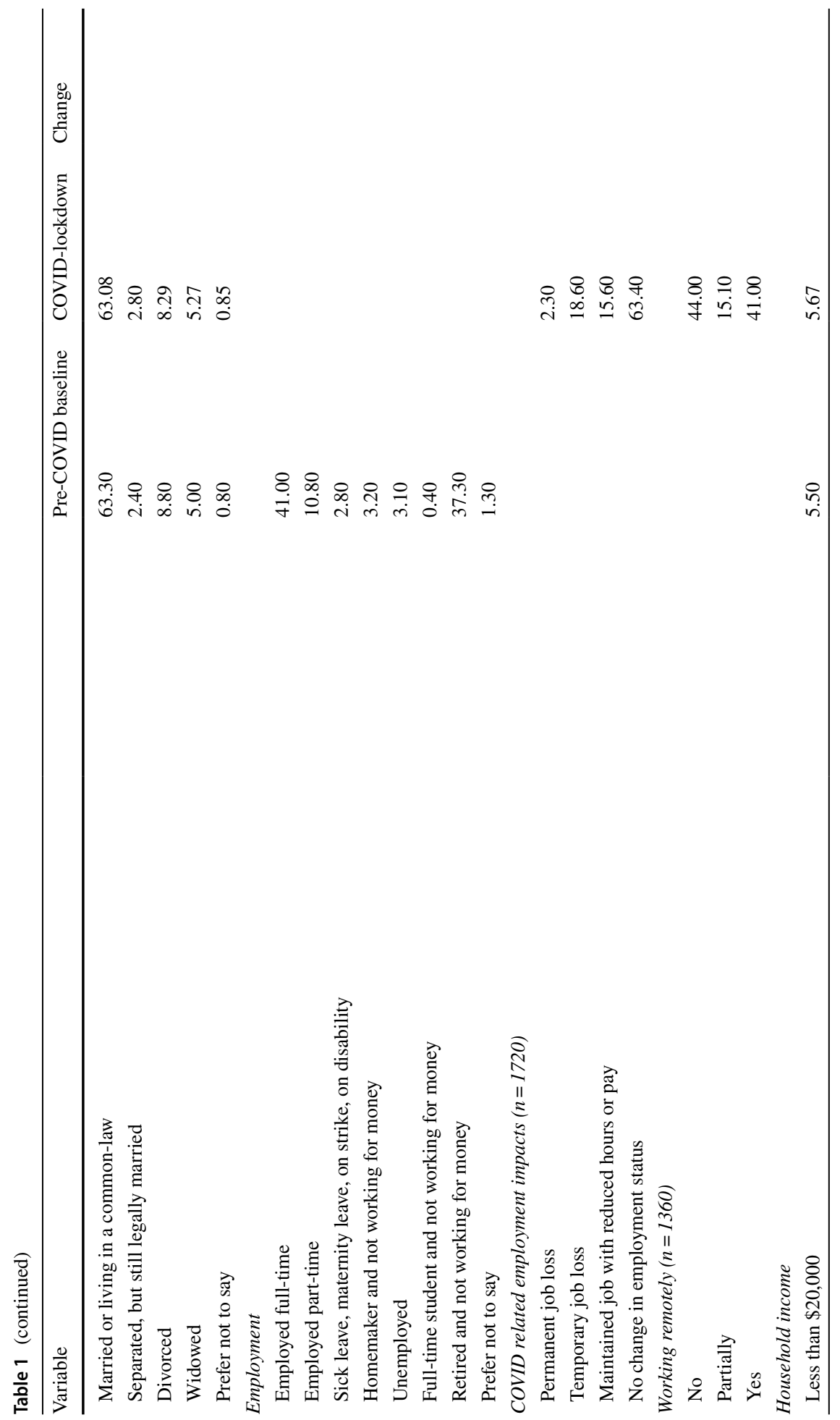




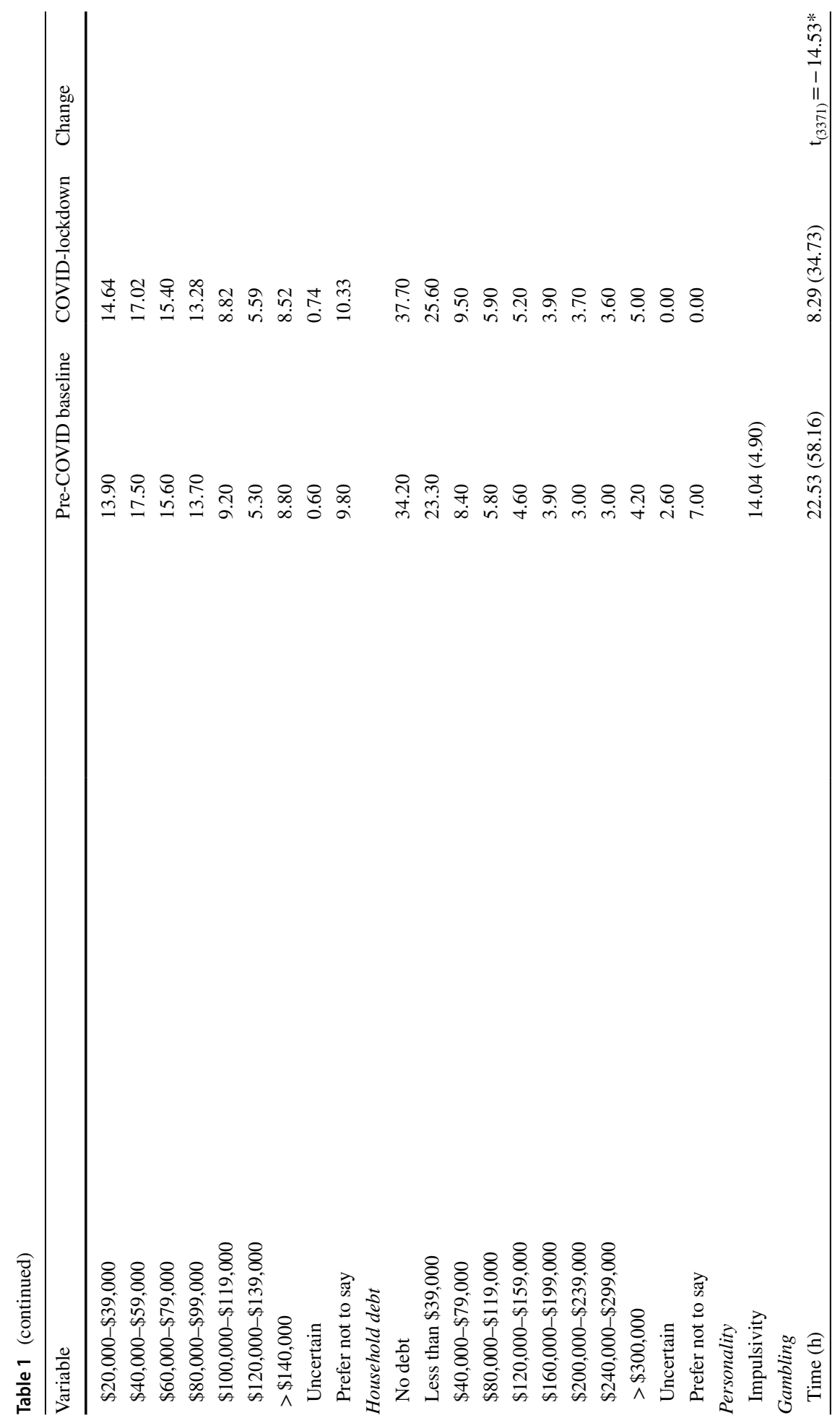




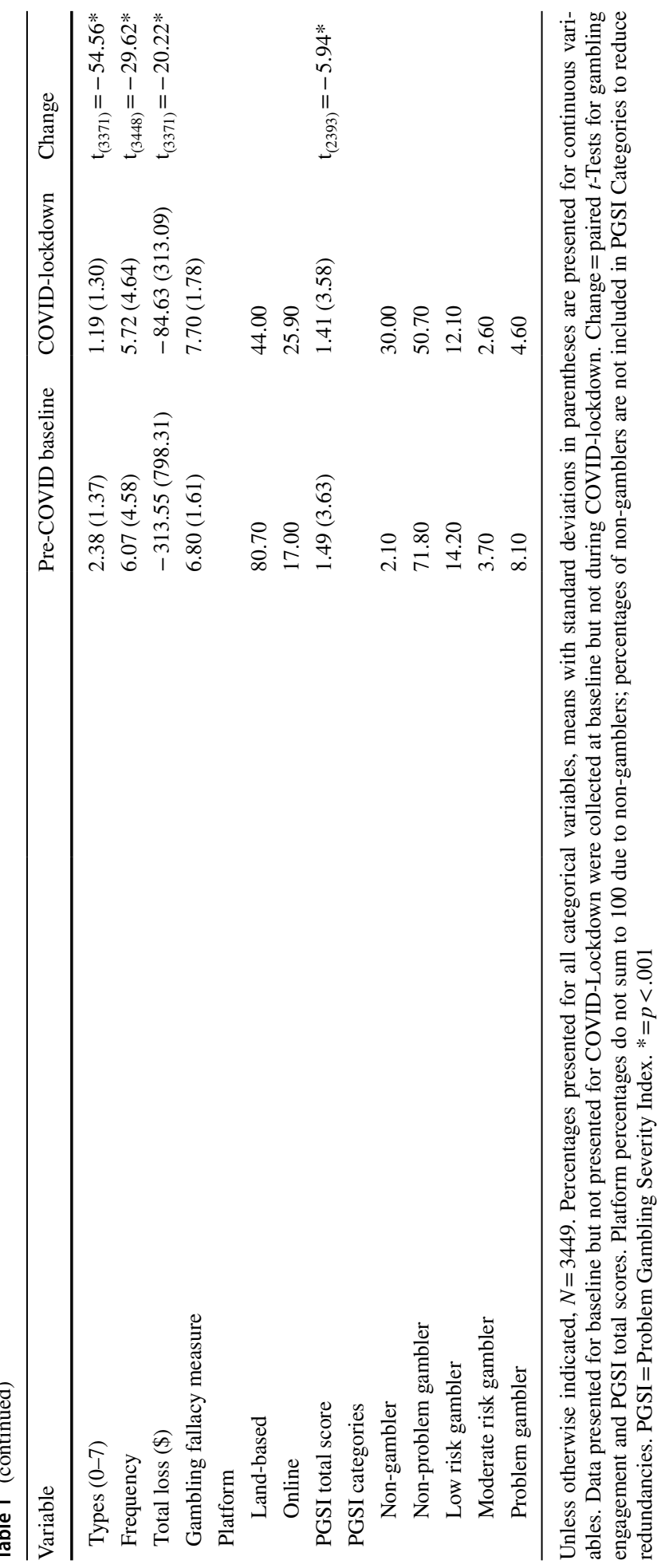


Table 2 Gambling engagement during lockdown as a function of perceived change

\begin{tabular}{llll}
\hline Change scores & \multicolumn{2}{l}{ Subjective reports of change in gambling frequency $(\mathrm{N}=2413)$} \\
\cline { 2 - 4 } & Decrease $(\mathrm{n}=574)$ & No Change $(\mathrm{n}=1460)$ & Increase $(\mathrm{n}=379)$ \\
\hline Gambling frequency & & $-1.10^{\mathrm{b}}$ & $0.48^{\mathrm{c}}$ \\
Mean & $-2.16^{\mathrm{a}}$ & 3.56 & 5.59 \\
SD & 3.81 & -1.00 & 0.00 \\
Median & -2.00 & 0.00 & 0.00 \\
Mode & -2.00 & & \\
Kruskal-Wallis & $\chi^{2}(2,2411)=110.64, p<.001, \eta^{2}=0.04$ & $-14.71^{\mathrm{b}}$ \\
Gambling time $(h)$ & & $-11.37^{\mathrm{b}}$ & 105.41 \\
Mean & $-19.00^{\mathrm{a}}$ & 45.94 & -2.00 \\
SD & 71.38 & -2.00 & -1.00 \\
Median & -4.00 & 0.00 & \\
Mode & 0.00 & & \\
Kruskal-Wallis & $\chi^{2}(2,2411)=17.53, p<.001, \eta^{2}=0.01$ & & \\
\hline
\end{tabular}

Change scores $\quad$ Subjective reports of change in gambling expenditure $(\mathrm{N}=2413)$

$\overline{\text { Decrease }(n=575) \quad \text { No Change }(n=1438) \quad \text { Increase }(n=400)}$

Total loss (\$)

$\begin{array}{llll}\text { Mean } & 282.70^{\mathrm{a}} & 247.66^{\mathrm{b}} & 425.12^{\mathrm{ab}} \\ \text { SD } & 739.56 & 698.97 & 1228.42 \\ \text { Median } & 58.00 & 36.50 & 56.53 \\ \text { Mode } & 0.00 & 0.00 & 0.00\end{array}$

Kruskal-Wallis

$\chi^{2}(2,2411)=14.60, p<.001, \eta^{2}=0.04$

Kruskal-Wallis test of between group differences. Bonferroni adjusted Mann-Whitney U post hoc test results indicated by superscript, where different superscript indicates statistically significant differences. PGSI = Problem Gambling Severity Index. Mean, standard deviation, median, and modal difference scores presented. Negative numbers indicate a decrease at lockdown, positive indicate an increase at lockdown

to a typical month prior to lockdown, gamblers gambled less frequently $\left(t_{(3448)}=-29.62\right.$, $\left.p=0.000, M_{D}=-2.06,95 \% \mathrm{CI}[-2.20,-1.93], d=0.45\right)$, gambled for significantly less time $\left(t_{(3371)}=-14.53, p=0.000, M_{D}=-14.82 \mathrm{~h}, 95 \% \mathrm{CI}[-16.82,-12.82], d=0.31\right)$, and had significantly lower total gambling-related financial losses $\left(t_{(3371)}=-20.22, p<0.001\right.$, $M_{D}=\$ 271.93,95 \%$ CI $\left.[-298.30,-245.57], d=0.47\right)$.

In terms of problem gambling, mean PGSI scores decreased significantly $\left(t_{(2393)}=-5.94, p=0.000, M_{D}=-0.32,95 \%\right.$ CI $\left.[-0.43,-0.21], d=0.09\right)$, and the proportion of the sample scoring in the problem gambling range decreased significantly from $7.00 \%$ to $4.60 \%(\mathrm{McNemar}=15.01, d f=2394, p<0.001)$. Yet, pre-COVID PGSI scores only account for $56.25 \%$ of the variance in COVID-Lockdown PGSI scores $(r=0.75)$ and as such, examination of other explanatory variables worthwhile. 


\section{Subjective Perception of Change in Gambling [G2]}

\section{Perceived Changes in Gambling}

Overall, $60.51 \%$ of the sample perceived their gambling frequency to be the same as it was prior to lockdown, with $23.78 \%$ reporting decreased gambling frequency, and $15.71 \%$ indicating that their gambling had increased. A Kruskal-Wallis test was used to examine whether pre-post COVID differences in gambling engagement (frequency and time spent gambling) were related to perceived change in gambling frequency (see Table 2). For this examination, COVID-Lockdown and pre-COVID difference scores were used. The Kruskal-Wallis test of total frequency showed significant differences between groups, $\chi^{2}(2$, 2413) $=110.64, p<0.001, \eta^{2}=0.04$. Bonferroni corrected post hoc Mann-Whitney U tests determined that the frequency of gambling engagement was significantly different for each group. Although the Mann-Whitney U tests are a test of ranks, these results make sense as the individuals perceiving a decrease showed a large median decrease, relative to individuals reporting no change who also showed a decrease, while individuals reporting an increase did not, in fact, show a median increase.

The Kruskal-Wallis test of time spent gambling also showed significant differences between groups, $\chi^{2}(2,2411)=17.53, p<0.001, \eta^{2}=0.01$. Post hoc analyses showed that the difference in time spent gambling for the 'decrease' group was significantly lower than the 'no change' and the 'increase' groups, but that the 'no change' and 'increase' groups did not differ from one another.

As noted, gambling related financial losses during lockdown were significantly lower than those during a typical month Pre-COVID. Yet only $23.83 \%$ of the sample report the perception of decreased gambling expenditures. A further $59.59 \%$ of the sample report no perceived change in gambling expenditures, and $16.58 \%$ indicate that their gambling expenditures had increased. The Kruskal-Wallis test of total gambling loss difference scores showed significant differences in the mean rank of groups, $\chi^{2}(2,2411)=14.60$, $p<0.001, \eta^{2}=0.004$. Post hoc tests showed that the changes in total losses differed significantly between the decrease and the no change groups, but no other group differences were detected.

\section{Profiles of Gamblers Who Increased Versus Decreased Gambling Expenditures and Frequency [G3]}

Cross-sectional examination of the profiles of individuals who had increased versus decreased their gambling frequency and expenditures during lockdown was undertaken using binary logistic regression. The models included any COVID-Lockdown variable that was associated with gambling change scores (frequency and expenditure respectively) at the 0.01 alpha level regardless of the strength of the association. Cross-sectional correlations can be seen in Table 3. Individuals' frequency change was classified according difference scores. Specifically, difference scores for gambling frequency were calculated and respondents with a positive difference score were classified as having 'increased' frequency, those with a negative difference score were classified as 'decreased' frequency, and those with a difference score equal to zero were classified as 'no change'. For changes in gambling expenditure, the absolute values of the net gambling expenditure variables were used. Gambling expenditures were classified as 'increased' if the absolute value of 


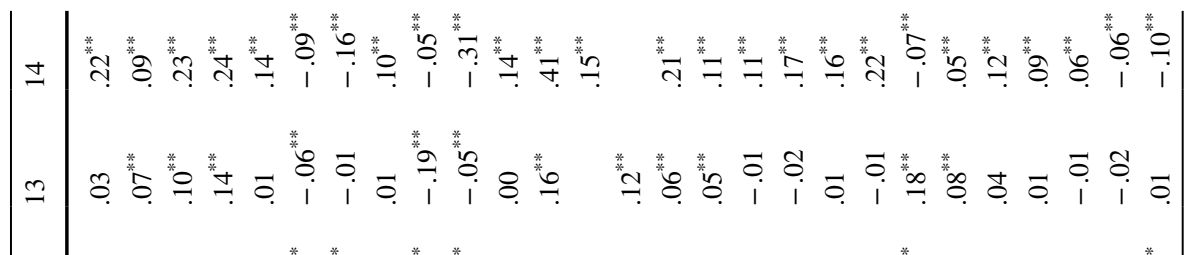

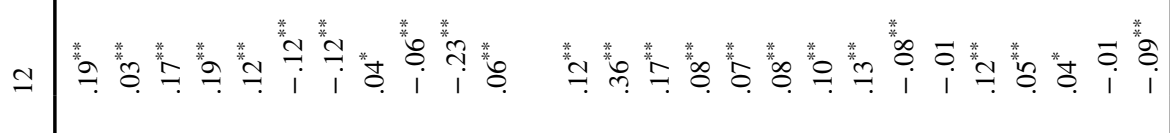
$=\left(\begin{array}{c}n \\ n_{*}\end{array}\right.$

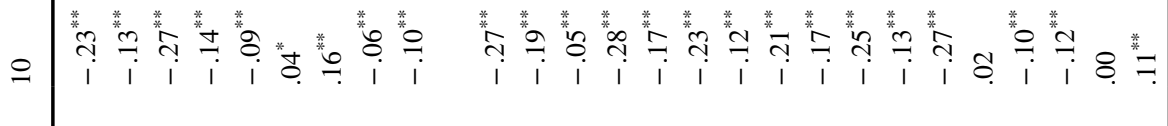

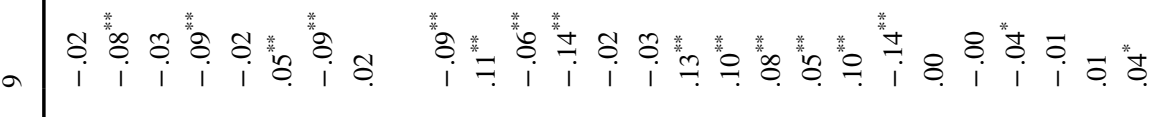

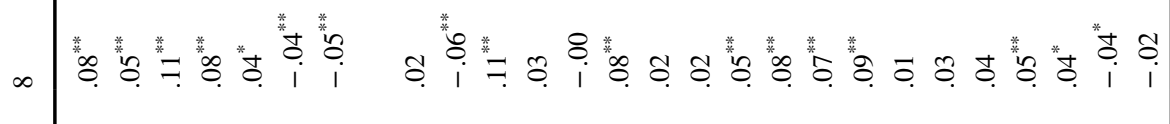

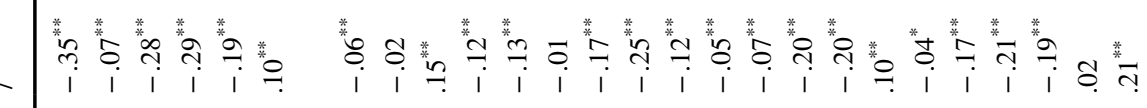

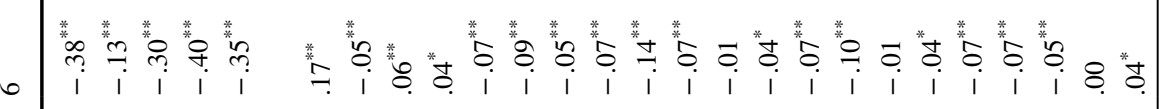
n

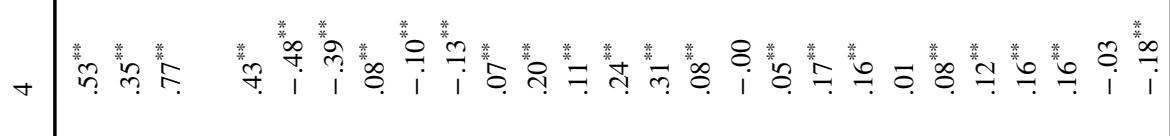

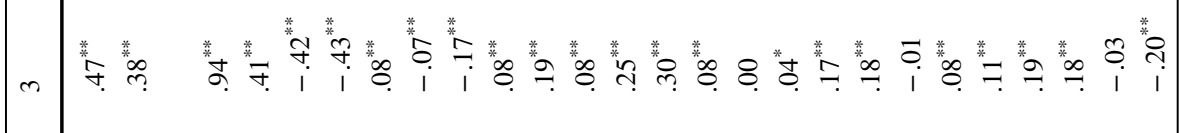
八 तै - •

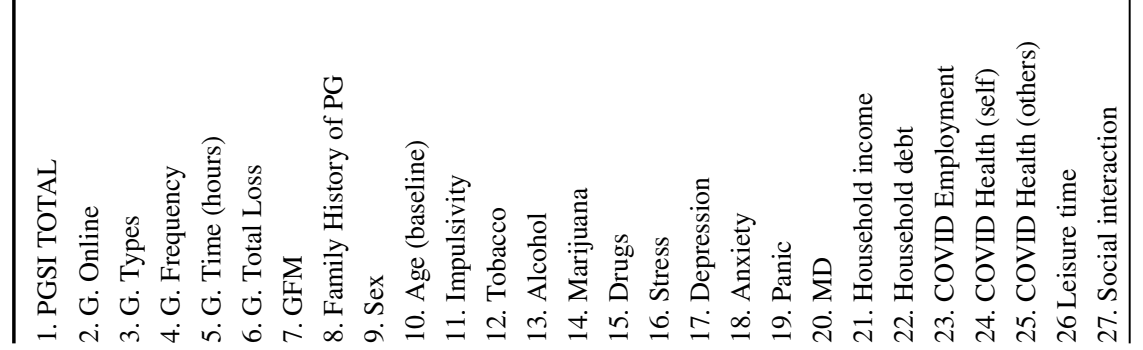




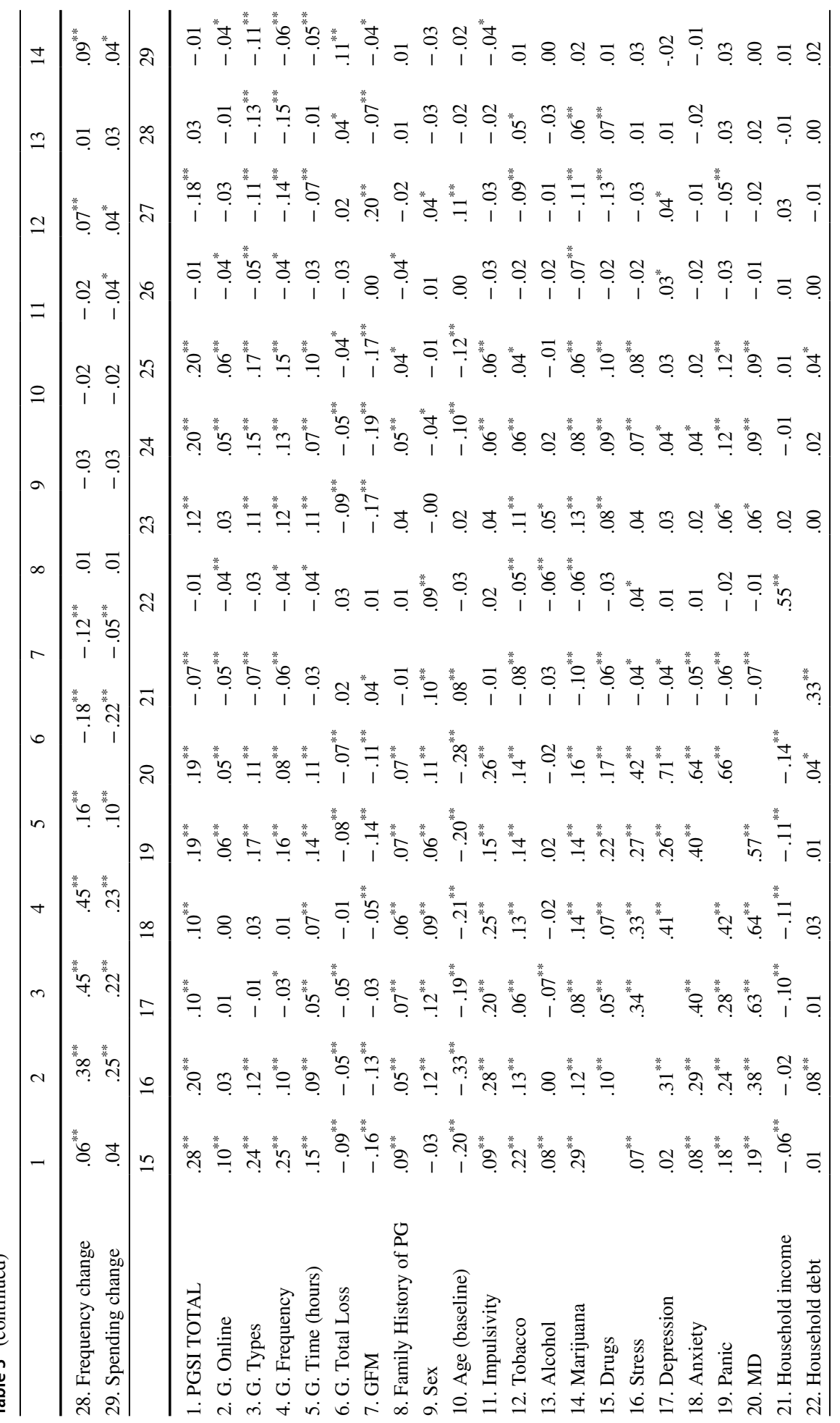




|


Table 4 Predicting increases versus decreases in gambling during the lockdown

\begin{tabular}{|c|c|c|c|c|c|}
\hline & \multirow[t]{2}{*}{ B } & \multirow[t]{2}{*}{ Wald } & \multirow[t]{2}{*}{ OR } & \multicolumn{2}{|l|}{$95 \% \mathrm{CI}$} \\
\hline & & & & Lower & Upper \\
\hline \multicolumn{6}{|l|}{ Frequency change } \\
\hline Gambling types & 0.90 & $184.28 * * *$ & 2.46 & 2.16 & 2.80 \\
\hline PGSI total & -0.15 & $44.95^{* * *}$ & 0.87 & 0.83 & 0.90 \\
\hline Mental health disorder & 0.30 & $4.56^{*}$ & 1.34 & 1.03 & 1.76 \\
\hline Constant & \multicolumn{5}{|c|}{$\chi^{2}(4,2961)=297.47, p<.001$} \\
\hline \multicolumn{6}{|l|}{ Expenditure change } \\
\hline Gambling frequency & 0.07 & $24.96^{* * *}$ & 1.07 & 1.04 & 1.10 \\
\hline Online gambling & 0.40 & $12.47 * * *$ & 1.49 & 1.19 & 1.86 \\
\hline PGSI total & -0.04 & $5.34 *$ & 0.96 & 0.93 & 0.99 \\
\hline Constant & \multicolumn{5}{|c|}{$\chi^{2}(3,3267)=56.34, p<.001$} \\
\hline
\end{tabular}

$\mathrm{B}=$ regression coefficient, Wald $=$ Wald statistic, $\mathrm{OR}=$ Odds ratio, $95 \%$ $\mathrm{CI}=95 \%$ Confidence Intervals of the Odds ratio, Lower and Upper bounds. PGSI $=$ Problem Gambling Severity Index. $* * *=p<.001$; $* *=p<.01 ; *=p<.05$

net expenditures was greater during lockdown than at pre-COVID baseline, 'decreased' if the value was less at COVID-Lockdown, and 'no change' if the values were equal.

After excluding individuals who were classified as having 'no change' in gambling frequency $(n=488)$ during the lockdown, data from 2961 respondents were included ( $n=2334$, decreased; $n=627$, increased). Binary logistic regression was conducted with 'increased frequency' as the reference group. Variable entry was forward stepwise with entry order determined by Wald magnitude of $p=0.10$ and removal at $p=0.15$, and a cut value of 0.30 . The test of the full model with four predictors against a constant only model was statistically significant, $\chi^{2}(4, N=2,961)=297.47, p<0.001$. The variance accounted for was moderate with Nagelkerke $R^{2}=0.20$. The overall prediction success of the model was moderate with $71.40 \%$ correctly classified, $66.30 \%$ of 'increased gambling' participants correctly classified and $73.70 \%$ of 'decreased gambling' participants correctly classified. Table 4 shows the regression coefficients, Wald statistics, odds ratios and $95 \% \mathrm{CI}$ confidence intervals for each of the predictor variables. In order of importance, the cross-sectional COVID-lockdown variables that best predicted increased gambling frequency during the spring lockdown were: engaging in more types of gambling and lower PGSI scores. The contribution of the mental disorder variable to the final model was significant at the 0.05 , but not at the 0.01 , alpha level.

A second binary logistic regression was conducted for the classification of those who increased $(n=479)$ versus decreased $(n=2788)$ gambling expenditures during the spring lockdown. Those who 'increased' expenditure were the reference group and variable entry was forward stepwise with entry order determined by a Wald magnitude of $p=0.10$, removal at $p=0.15$ and a cut value of 0.20 . The test of the full model against a constant only model was statistically significant, $\chi^{2}(3, N=3,267)=56.34, p<0.001$, but the variance accounted for was small with Nagelkerke $R^{2}=0.04$. The overall prediction success of the model was moderate with $60.80 \%$ correctly classified, $56.20 \%$ of 
Table 5 Responses to COVID health experience questionnaire

\begin{tabular}{lll}
\hline & Self & $\begin{array}{l}\text { Other(s) in } \\
\text { household }\end{array}$ \\
\hline Never had symptoms, never was tested & 3140 & 2216 \\
Never had symptoms, was tested, waiting for results & 242 & 157 \\
Never had symptoms, was tested, tested negative & 246 & 163 \\
Never had symptoms, was tested, tested positive & 183 & 121 \\
Previously had symptoms, do not have symptoms now, was not tested & 257 & 148 \\
Previously had symptoms, do not have symptoms now, was tested, tested negative & 159 & 93 \\
Previously had symptoms, do not have symptoms now, was tested, tested positive & 106 & 67 \\
Currently have symptoms, was not tested & 107 & 69 \\
Currently have symptoms, was tested, waiting for results & 99 & 63 \\
Currently have symptoms, was tested, tested negative & 100 & 65 \\
Currently have symptoms, was tested, tested positive & 95 & 63 \\
Had medical treatment due to severe symptoms & 18 & 11 \\
Hospital stay due to COVID-19 & 13 & 9 \\
House mate died of COVID-19 & & 9 \\
Close family or friend died of COVID-19 (non-housemate) & & 115 \\
\hline
\end{tabular}

Frequency of responses to questionnaire item options indicating experience with the COVID virus

'increased gambling' participants correctly classified and $62.00 \%$ of 'decreased gambling' participants correctly classified. Table 4 also shows the regression results for the of those who increased gambling expenditures. According to Wald criterion, increased gambling frequency and engaging in online gambling reliably predicted an increase in gambling expenditures during lockdown. PGSI scores contribute significantly to this model at the 0.05 level, but not at our a priori alpha level.

\section{Predicting PGSI Gambling Category During Lockdown [G4]}

The final planned analysis examined predictors of PGSI gambling category during the COVID lockdown, including COVID health and employment impacts. COVID pandemic employment specific impacts included whether respondents had lost their job, temporarily or permanently, due to the pandemic or if their work hours had been reduced. Six hundred and twenty-nine respondents had pandemic related employment impacts, with $20.90 \%$ having lost a job-2.30\% permanently and $18.60 \%$ temporarily-and $41 \%$ of the employed sample reporting working remotely due to the pandemic. Table 1 displays the frequency of COVID employment impacts along with pre-pandemic employment status for point of reference. For the purpose of analysis, indices of COVID impacts on personal health and the health of others included having virus-related symptoms, whether untested or tested, tested and waiting results or tested COVID confirmed, but excluding those who had symptoms but tested negative. Indices of COVID health impacts also included receiving medical 
Table 6 Concurrent GEE model predicting PGSI categories during lockdown

\begin{tabular}{llll}
\hline & Wald Chi-Square & df & Sig \\
\hline (Intercept) & 117.393 & 1 & .00 \\
Gambling online & 1212.354 & 2 & .00 \\
Gambling fallacies & 67.940 & 1 & .00 \\
Total gambling losses & 46.681 & 1 & .00 \\
Impulsivity & 38.105 & 1 & .00 \\
Gambling frequency & 26.336 & 1 & .00 \\
Tobacco use & 20.235 & 7 & .01 \\
Age & 16.143 & 1 & .00 \\
Time spent gambling & 14.967 & 1 & .00 \\
Data collection period & 11.643 & 1 & .00 \\
\# Of types of gambling games & 9.485 & 1 & .00 \\
Stress & 7.943 & 1 & .01 \\
Marijuana use & 4.782 & 7 & .69 \\
Social interaction & 3.903 & 1 & .05 \\
Illicit drug use & 3.755 & 1 & .05 \\
COVID—self health & 3.076 & 1 & .08 \\
COVID—employment impacts & 1.508 & 1 & .22 \\
Mood disorder & .224 & 1 & .64 \\
COVID—other's health & .006 & 1 & .94 \\
\hline Concurent GE & & &
\end{tabular}

Concurrent GEE output $(n=1666)$ ordered by resultant Wald score after Intercept. $\mathrm{Df}=$ degrees of freedom. Sig=obtained significance level

treatment and/or hospitalization for self or others, and for the 'others health' variable, death due to COVID. Table 5 presents the frequency of responses to all health-related experience questions.

The $\mathrm{GEE}^{2}$ model included any Pre-COVID or COVID-Lockdown variable that was associated with PGSI scores provided it was moderate to strongly associated and significant at our a priori 0.01 alpha level. Recall that Table 3 presents the univariate associations of all variables included in this study for both data collection periods. The composite mental health disorder variable was included instead of including depression, anxiety, and panic disorder separately. A 'Year' variable was included to account for the inter-assessment correlations and repeated assessment effects such as practice or learning. COVID specific health and employment index variables were also included, as was the variable assessing perceived lockdown related changes in the amount of social interaction.

The full test of model effects is presented in Table 6. The resultant significant predictor variables, in accordance with the magnitude of the Wald statistic were: online gambling (Wald $=1212.35, p<0.001$ ), gambling fallacies (Wald $=67.94, p<0.001$ ), total gambling losses (Wald $=46.68, p<0.001$ ), impulsivity (Wald $=38.11, p<0.001$ ), gambling

\footnotetext{
${ }^{2}$ Pre-COVID baseline and COVID-Lockdown variables differ slightly in respect to time reference: a typical month vs the past month. Given this variation, study goal 4 could have been addressed via regression, however, GEE was deemed more appropriate as it prevents the artificial inflation of variance explained.
} 
frequency on all types of gambling (Wald $=26.34, p<0.001$ ), use of tobacco products (Wald $=20.24, p<0.01$ ), younger age (Wald $=16.14, p<0.001)$, time spent gambling $($ Wald $=14.97, p<0.001)$, year $($ Wald $=11.64, p<0.001)$, stress $($ Wald $=7.94, p<0.01)$, number of types of gambling engaged in (Wald $=9.49, p<0.01)$. The following variables were significant at the 0.05 alpha level but are not significant at the a priori 0.01 alpha level: decreased social interaction since lockdown (Wald $=3.90, p<0.05$ ) and illicit drug use $($ Wald $=3.76, p<0.05)$.

\section{Discussion}

The novel COVID virus, and the social distancing responses to it, have impacted world citizens both socially and economically. The period of social and economic lockdown, a social distancing response, introduced the forced abstinence from legal in-person landbased gambling opportunities whilst online gambling opportunities continued. This situation, which was a historical first, led to much speculation about potential changes in gambling world-wide (e.g., Elder, 2020; Howie, 2020; Shivdas, 2020; Turner, 2020). The current study was uniquely positioned to examine pandemic related changes in gambling in Canada for two reasons. First, this study had quantifiable baseline gambling behavioral data that were collected from this sample six month prior to the lockdown in Canada, and second, data collection for the lockdown period began one month after lockdown was instituted in all provinces and concluded prior to any legal land-based establishment re-opening. Behavioral reports, therefore, were specific to the time of lockdown for all participants. Within this locked-down context, the current investigation aimed to address four key research goals.

The first goal was to examine changes in individuals' gambling involvement. Overall, although most continued to gamble during lockdown, almost a third of gamblers ceased gambling altogether. Among those who continued to gamble, significant decreases were detected on all gambling engagement measures: number of gambling types engaged in, gambling frequency, time spent in gambling sessions, as well as a significant reduction in gambling related financial losses. These reductions parallel those found in other surveys in Canada and elsewhere (Hodgins \& Stevens, 2021). In light of the fact that the majority of the sample at baseline report engaging in gambling activities via landbased only platforms these decreases make intuitive sense-gambling engagement will decrease while legal land-based platforms are restricted or entirely inaccessible. It will be important to study these individuals longitudinally to see whether they re-engage in gambling over time as gambling opportunities return and whether this is associated with their problem gambling status. Some have speculated that problem gamblers may use the lockdown as a means with which to curtail their gambling problem (e.g., Elder, 2020).

Gambling platforms were examined for changes in gambling involvement. During the lockdown $17.60 \%(n=490)$ of pre-pandemic Land-based only gamblers migrated to the online gambling platform. Migration was a concern as online gambling has been associated with greater problem gambling (e.g., Håkansson et al., 2020; Wood \& Williams, 2009, 2011; Xuereb et al., 2020). Migrators, together with pre-pandemic online gamblers, comprised over one third of the Pre-COVID sample. Yet, problem gambling scores-irrespective of gambling platform-were significantly lower during lockdown. These scores, when examined according to gambler type category, yield evidence that 
the proportion of gamblers in the sample that were classified as problem gamblers was markedly lower during lockdown. Future studies should seek to identify the characteristic profile and gambling motivations of migrators compared to those of regular online gamblers. Xuereb et al., (2020) found that migrators tended towards greater problem gambling severity relative to land-based gamblers. It remains unexplored whether migrators differ from regular (pre-pandemic) online gamblers in level of gambling problems or other characteristics as this examination was beyond the scope of the current study. Nonetheless, subsequent research is also necessary to establish whether migrators retain online gambling in their repertoire once land-based options re-emerge. Attention should be given to the profiles of migrators who do, versus those who do not, continue to gamble online as this may be informative for future investigations and interventions.

The second aim of this study had two components: to examine individuals' perception of changes in gambling engagement during lockdown and then to examine quantifiable differences in gambling behavior as a function of these perceived changes. Over half of the gambling sample indicated that their gambling engagement, frequency and expenditures, had not changed during the lockdown. So, for the majority of gamblers, lockdown appears to have had little or no influence. Perception of change among the remaining gamblers was split, with just over half indicating perceived decreases in their gambling and just under half indicating perceived increases in their gambling frequency and expenditures.

Quantifiable differences in gambling engagement were somewhat consistent with perceived changes in that individuals who reported a perceived decrease also showed greater actual change in frequency, time, and financial losses compared to individuals who reported no change in their gambling. The group reporting a perceived increase, on the other hand, did not consistently differ from the other perceived change groups. They did report a small mean increase in frequency, however the median change for this group was zero, and they did not differ from the no perceived change group in gambling time or expenditure. The lack of consistent, and in some cases significant, differences illustrate the advantages of relying on changes in behavior assessed longitudinally instead of loosely defined selfreported perception of change. That having been said, establishing a baseline indicator of gambling is not straight forward. In this case, we used gambling in a typical month in the year prior aggregated over different types of gambling, which seems like a good overall indicator of gambling engagement.

This study also aimed to identify the individual differences that discriminate between gamblers who had quantifiable increases versus decreases in gambling frequency and expenditure. Gamblers who actually increased engagement frequency during the pandemic Lockdown in March were those who engaged with more types of games but had lower PGSI scores. Thus, it appears that those who increased gambling engagement during the spring lockdown were predominantly recreational gamblers with a diverse game repertoire. Reports of greater mental health issues in association with pandemic period gambling were a significant correlate of increased gambling frequency and therefore should be monitored in subsequent studies. The predictors that delineated gamblers with increases versus decreases in gambling expenditures did not overlap with those for frequency. Greater gambling frequency and engaging in online gambling reliably discriminated between those with expenditure increases versus decreases. The modest level of classification and variance explained for those who increased gambling during lockdown, suggests that the characteristic profile of this subset of gamblers is diverse. Nonetheless, that the factors identified as significant predictors are those that are typically related to problem gambling (Allami et al., 2021) suggests that individuals who increased gambling during lockdown may be at increased risk of subsequent gambling problems. COVID-specific factors, such 
as health and employment impacts, were not significant predictors of changes in gambling. They were, however, significantly associated with problem gambling scores. When coupled with the recognition that the lockdown was part of a swift Canadian pandemic response, this suggests that the full impact of the COVID pandemic on gambling is yet unknown. COVID-specific health, economic and social factors should therefore remain a research focus.

The final goal of this study was to identify variables that predicted gambler type/problem gambling category. The results of the concurrent GEE model, which took into account changes over time and COVID specific impacts (health, economic and social), identified multiple predictive variables: online gambling, gambling fallacies, total gambling losses, impulsivity, gambling frequency on all types of gambling, use of tobacco products, younger age, time spent gambling, year (accounting for changes over time), stress, and types of gambling engaged in. These results are consistent with the biopsychosocial model of problem gambling that posits multiple risk factors in the etiology of problem gambling and with the results of previous large scale studies testing the model (Billi et al., 2014; elGuebaly et al., 2015; Romild et al., 2014; Williams et al., 2015). In addition, these results support the conclusion that problem gambling treatments and interventions both require a broad assortment of policy and educational initiatives to be efficacious (see for example: Christensen, 2018; Williams et al., 2012; Williams \& Volberg, 2010). That being said, future examinations should continue to monitor COVID specific variables for their impact on gambling and problem gambling. Data collection for this study, for example, took place early in the first phase of the pandemic. While the short-term impacts, beyond stress, did not significantly predict gambling category the long-term impacts may be more deleterious.

This study illustrates the value of prospective, longitudinal data, when considering for example, the mismatch between participants perceived change in their gambling and the data collected before and during the pandemic. One limitation is that all the data available, subjective assessment of change and quantitative values, are self-reported. The most rigorous self-report measures available were used, but self-report is still subject to various biases. It was helpful that the sample of regular gamblers was large and diverse in terms of geography, demographic characteristics, and gambling engagement. However, it was collected via an online panel which might have implications for the willingness of participants to gamble online. It is also possible that this sample was not necessarily representative of all gamblers in other ways as there was significant attrition between the first assessment (not included in these analyses) and the second assessment (this Pre-COVID sample). These limitations will be more fully addressed when the next wave of data are available for analysis (i.e., late 2020). Moreover, the next assessment will allow for continuity in the examination of the COVID pandemic on gambling in Canada.

\section{Conclusion}

The current study aimed to investigate the impact of the COVID pandemic lockdown on gambling and problem gambling in Canada. Re-surveying AGRI National Project's online panel participants $(n=3449)$ during the Canadian lockdown allowed for the quantitative comparison of gambling during this period relative to typical gambling behaviors six months prior to the onset of the pandemic. This study found that nearly one-third of gamblers ceased gambling during the lockdown altogether. Among those who remained gambling, quantitative data indicates that gambling decreased across nearly all engagement 
metrics including significant decreases in: gambling frequency, time spent in gambling sessions, money spent, and the number of game types engaged in. Qualitative perceptions of changes in gambling were examined and, while some differences in gambling engagement were found between groups, the accuracy of qualitative reports of change were found to be questionable. Gambling platform was the only gambling engagement metric where increases were found. Over $17 \%$ of the gambling sample migrated from pre-pandemic landbased only to online gambling during the lockdown, bringing the total sample participation in online gambling up to nearly $50 \%$. While it was found that problem gambling within the sample had generally reduced, consistent with previous literature, it was also found that gambling online - among other biopsychosocial factors-was a significant predictor for classification as a problem gambler during the lockdown. Subsequent studies are therefore necessary to examine the future experiences and engagement trajectories of migrators specifically. As online gambling was found to be a risk factor for problem gambling, migrators may have inadvertently increased their risk. Future examination will reveal if this risk manifests in subsequent gambling problems. Future studies are also required more generally, to assess if the pandemic related changes in gambling detected herein remain stable, or if engagement reverts to pre-pandemic levels when the pandemic response allows for the re-opening of land-based gambling venues.

Acknowledgements This research was conducted with funding assistance from the Alberta Gambling Research Institute to Carrie Shaw and David Hodgins, and with funding assistance from the AGRI National Project (ANP), a collaborative national project, which received funding from the Alberta Gambling Research Institute, the Canadian Consortium for Gambling Research, the Canadian Centre for Substance Abuse and Addiction, and the Gambling Research Exchange Ontario.

\section{Declarations}

Conflict of interest The authors declare no conflict of interest that would impact the current study content, conclusions, or presentation.

\section{References}

Abel, T., \& McQueen, D. (2020). The COVID-19 pandemic calls for spatial distancing and social closeness: Not for social distancing! International Journal of Public Health, 65(3), 231-231. https://doi.org/10. 1007/s00038-020-01366-7

Allami, Y., Hodgins, D. C., Young, M., Brunelle, N., Currie, S., Dufour, M., Flores-Pajot, M.-C., \& Nadeau, L. (2021). A meta-analysis of problem gambling risk factors in the general adult population. Addiction. https://doi.org/10.1111/add.15449

American Psychiatric Association, American Psychiatric Association, \& DSM-5 Task Force. (2013). Diagnostic and statistical manual of mental disorders: DSM-5. American Psychiatric Association.

Auer, M., Malischnig, D., \& Griffiths, M. D. (2020). Gambling before and during the COVID-19 pandemic among European regular sports bettors: An empirical study using behavioral tracking data. International Journal of Mental Health and Addiction. https://doi.org/10.1007/s11469-020-00327-8

Biddle, N. (2020). Gambling during the COVID-19 pandemic. https://csrm.cass.anu.edu.au/sites/default/ files/docs/2020/12/Gambling_during_the_COVID-19_pandemic.pdf

Billi, R., Stone, C. A., Marden, P., \& Yeung, K. (2014). Victorian gambling study: A longitudinal study of gambling and health in Victoria, 2008-2011. Victorian Responsible Gambling Foundation.

Brunelle, N., Leclerc, D., Cousineau, M., Dufour, M., Gendron, A., \& Martin, I. (2012). Internet gambling, substance use, and delinquent behavior: An adolescent deviant behavior involvement pattern. Psychology of Addictive Behaviors, 26(2), 364-370. https://doi.org/10.1037/a0027079

Campbell, C. (2020). Coronavirus: Lockdown leaves addicts "close to relpase". BBC News. https://www. bbc.com/news/uk-northern-ireland-52811931 
Christensen, D. R. (2018). Effectiveness of prevention strategies. In Preventing Gambling Addiction. Routledge: Centre for Excessive Gambling (Centre du Jeu Excessif: CJE), Switzerland, \& NHS National Problem Gambling Clinic, and Imperial College.

Costa, P. T., \& McCrae, R. R. (1992). NEO PI-R. Psychological Assessment Resources Inc.

Czegledy, P. (2020). Canadian land-based gambling in the time of COVID-19. Gaming Law Review, 24(8), 555-558. https://doi.org/10.1089/glr2.2020.24811

Economou, M., Souliotis, K., Malliori, M., Peppou, L. E., Kontoangelos, K., Lazaratou, H., Anagnostopoulos, D., Golna, C., Dimitriadis, G., Papadimitriou, G., \& Papageorgiou, C. (2019). Problem gambling in Greece: Prevalence and risk factors during the financial crisis. Journal of Gambling Studies, 35(4), 1193-1210. https://doi.org/10.1007/s10899-019-09843-2

el-Guebaly, N., Casey, D. M., Currie, S. R., Hodgins, D. C., Schopflocher, D. P., Smith, G. J., \& Williams, R. J. (2015). The leisure, lifestyle, and lifecycle project (LLLP): A longitudinal study of gambling in Alberta (pp. 1-161) [Final Report prepared for the Alberta Gambling Research Institute]. Alberta Gaming Research Institute.

Elder, J. (2020). Tim Costello on COVID-19: A wake-up call for fathers, a reprieve for gamblers. Thew New Daily. Retrieved from https://thenewdaily.com.au/news/people/2020/05/16/tim-coste llo-and-covid-19/

Ferris, J., \& Wynne, H. (2001). The Canadian problem gambling index: User Manual.

Fluharty, M., Paul, E., \& Fancourt, D. (2020). Predictors and patterns of gambling behaviour across the COVID-19 lockdown: Findings from a UK cohort study Retrieved January 26 2021, from https:// doi.org/10.31234/osf.io/8qthw

Grasso, D. G., Briggs-Gowan, M. J., Ford, J. D., \& Carter, A. S. (2020). The Epidemic-Pandemic Impacts Inventory (EPII).

Gunstrone, B., Gosschalk, K., Joyner, O., Diaconu, A., \& Skeikh, M. (2020). The impact of the COVID19 lockdown on gambling behaviour, harms, and demand for treatment and support. YouGov. https://about.gambleaware.org/media/2284/yougov-covid-19-report.pdf

Håkansson, A., Fernández-Aranda, F., Menchón, J. M., Potenza, M. N., \& Jiménez-Murcia, S. (2020). Gambling during the COVID-19 crisis-A cause for concern? Journal of Addiction Medicine. https://doi.org/10.1097/ADM.0000000000000690

Hemphill, J. F. (2003). Interpreting the magnitudes of correlation coefficients. American Psychologist, 58(1), 78-79. https://doi.org/10.1037/0003-066X.58.1.78

Horner, A. (2020). Online casinos searchers a "all-time high" during lockdown. BBC News. https://www. bbc.com/news/uk-england-52633355

Hodgins, D. C., \& Stevens, R. M. G. (2021). The impact of COVID-19 on gambling and gambling disorder: Emerging data. Current Opinion in Psychiatry, 34(4), 332-343. https://doi.org/10.1097/YCO. 0000000000000709

Howie. (2020). Covid 19 coronavirus: Lotto feels lockdown crunch with fewer tickets bought-NZ Herald. https://www.nzherald.co.nz/nz/news/article.cfm?c_id=1\&objectid=12332257

International Gambling Business. (2020). GC Survey: Gambling in decline under lockdown. International Gambling Business. https://www.igamingbusiness.com/news/gc-survey-gambling-declineunder-lockdown

Jones, V. (2020). Ex gambling addict who lost more than 700,000. Pounds says lockdown has created the "perfect storm" for relapse. Nottinghamshire LIve. https://www.nottinghampost.com/news/ukworld-news/ex-gambling-addict-who-lost-4126289

Leonard Shaw, C. A., Williams, R. J., \& McGrath, D. S. (2020). Gambling fallacies: Predicting problem gambling in a national sample. Psychology of Addictive Behaviors. https://doi.org/10.1037/adb00 00673

Leonard Shaw, C. A., Williams, R. J., \& Vokey, J. (2015). Gambling fallacies: What are they and how are they best measured? Journal of Addiction Research and Therapy. https://doi.org/10.4172/21556105.1000256

Marsden, J., Darke, S., Hall, W., Hickman, M., Holmes, J., Humphreys, K., Neale, J., Tucker, J., \& West, R. (2020). Mitigating and learning from the impact of COVID-19 infection on addictive disorders. Addiction, 115(6), 1007-1010. https://doi.org/10.1111/add.15080

Moraine, J. (2020). GVC praises additional guidance by UKGC in response to lockdown. Gambling News. https://www.gamblingnews.com/news/gvc-praises-regulatory-measures-by-ukgc-in-respo nses-to-lockdown/

Olason, D. T., Hayer, T., Meyer, G., \& Brosowski, T. (2017). Economic recession affects gambling participation but not problematic gambling: Results from a population-based follow-up study. Frontiers in Psychology, 8, 1247. https://doi.org/10.3389/fpsyg.2017.01247 
Romild, U., Volberg, R., \& Abbott, M. (2014). The Swedish Longitudinal Gambling Study (Swelogs): Design and methods of the epidemiological (EP-) track. International Journal of Methods in Psychiatric Research, 23(3), 372-386. https://doi.org/10.1002/mpr.1449

Shivdas, S. (2020). Shut casinos hit Caesars as COVID-19 puts gambling industry in survival mode. Reuters. https://www.reuters.com/article/us-caesars-results-idUSKBN22N2SJ

Turner, N. E. (2020). COVID-19 and gambling in Ontario. Journal of Gambling Issues. https://doi.org/ 10.4309/jgi.2020.44.1

van Schalkwyk, M. C. I., Cheetham, D., Reeves, A., \& Petticrew, M. (2020). Covid-19: We must take urgent action to avoid an increase in problem gambling and gambling related harms. The BMJ. https://blogs. bmj.com/bmj/2020/04/06/covid-19-we-must-take-urgent-action-to-avoid-an-increase-in-problemgambling-and-gambling-related-harms/

Williams, R. J., Hann, R., Schopflocher, D., West, B., McLaughlin, P., White, N., King, K., \& Flexhaug, T. (2015). Quinte longitudinal study of gambling and problem gambling [Technical Report]. Ontario Problem Gambling Research Centre. http://www.uleth.ca/dspace/handle/10133/3641

Williams, R. J., \& Volberg, R. A. (2010). Best practices in the populations assessment of problem gambling. (pp. 1-97). Ontario Problem Gambling Research Center.

Williams, R. J., Volberg, R. A., Stevens, R. M. G., Williams, L. A., \& Arthur, J. N. (2017). The definition, dimensionalization, and assessment of gambling participation. Report for the Canadian Consortium for Gambling Research. https://www.uleth.ca/dspace/handle/10133/4838

Williams, R. J., West, B. L., \& Simpson, R. I. (2012). Prevention of Problem Gambling: A comprehensive review of the evidence, and identified best practices. Report Prepared for the Ontario Problem Gambling Research Centre and the Ontario Ministry of Health and Long Term Care.

Wood, R. T., \& Williams, R. J. (2007). 'How much money do you spend on Gambling?' The comparative validity of question wordings used to assess gambling expenditure. International Journal of Social Research Methodology, 10(1), 63-77. https://doi.org/10.1080/13645570701211209

Wood, R. T., \& Williams, R. J. (2009). Internet gambling: Prevalence, patterns, problems, and policy options (pp. 1-152) [Final Report prepared for the Ontario Problem Gambling Research Center]. Ontario Problem Gambling Research Center. http://hdl.handle.net/10133/693

Wood, R. T., \& Williams, R. J. (2011). A comparative profile of the Internet gambler: Demographic characteristics, game-play patterns, and problem gambling status. New Media and Society, 13(7), 1123-1141. https://doi.org/10.1177/1461444810397650

Xuereb, S., Kim, H. S., Clark, L., \& Wohl, M. J. A. (2020). Substitution behaviors among casino gamblers during COVID-19 precipitated casino closures. Preprint.

Publisher's Note Springer Nature remains neutral with regard to jurisdictional claims in published maps and institutional affiliations.

\section{Authors and Affiliations}

\section{Carrie A. Shaw ${ }^{1,2}$ D David C. Hodgins ${ }^{3}$ D $\cdot$ Robert J. Williams ${ }^{2}$ D $\cdot$ Yale D. Belanger $^{2}$ (D) Darren R. Christensen ${ }^{2}$ (D) Nady el-Guebaly ${ }^{3}$ (D) Daniel S. McGrath ${ }^{3}$ (D) Fiona Nicoll ${ }^{4}$. Garry J. Smith ${ }^{4} \cdot$ Rhys M. G. Stevens ${ }^{2} \mathbb{D}$}

1 Centre of Excellence in Responsible Gaming, University of Gibraltar, Europa Point Campus, Gibraltar GX11 1AA, Gibraltar

2 University of Lethbridge, Lethbridge, Canada

3 University of Calgary, Calgary, Canada

4 University of Alberta, Edmonton, Canada 